Palliative Care Team held an extraordinary multi-disciplinary team meeting to reflect on the previous year.

We gave space and time to discuss the care and support we have provided to specific individuals who have had a personal impact on us as human beings and professionals working in extremely challenging circumstances. Each discipline from within the multi-disciplinary team was invited to present at least one case, and discuss this person's story, and their own story within this, to consider the impact this person and their care had for them, and their learning from this. Within this meeting we heard contributions from nurses, doctors, arts therapists, social workers, the hospice chaplain, a cognitive behavioural therapist, and the rehabilitative allied health professionals.

In this presentation, by layering the St Christopher's Lantern Model of Palliative and End of Life Care Nursing onto each story, we present a number of the case studies we discussed, which highlight the unique contribution of palliative care professionals at a unique point in the history of a global pandemic. The Lantern Model (Mitchell, 2021; Overview of the Lantern Model - see: https://cdn.ps.emap.com/wp-content/ uploads/sites/3/2021/03/Overview_of_the_Lantern_Model.pdf)

supports practitioners to be curious in relation to the key tenets that shape and guide the care being provided, the context of care, the personal prerequisites required to provide care, the organisational conditions required to support care, the processes of nursing care, and the possible outcomes of care. Therefore, we will draw on the components of the Lantern Model to highlight and celebrate the work of a specialist palliative care team at a unique time in history.

\section{0-4 HIDDEN WORK AND HIDDEN WORKERS IN HOSPICE CARE}

Natalie Richardson. University of York, York, UK

10.1136/spcare-2021-Hospice.4

In this presentation, I draw on an ethnographic study conducted as part of a $\mathrm{PhD}$ research project in a hospice in England over a six-month period. The research explored the everyday working lives of a range of hospice workers, from clinical staff to cooks and complementary therapists. I will explore the hidden work of those in the spaces of the hospice often unexplored, such as in the kitchens and the therapy spaces. In these places, intimate interactions unfold and moments of sensory care between patients and staff take place. Here, I particularly focus on the hospice workers' influence and involvement in experiences at the end of life through the three sensory domains of silence, touch and taste. The use of silence and touch have been well explored in hospice settings, however, this paper adds the importance of those in often hidden spaces, carrying out hidden sensory forms of care. The touch of a cleaner on a patient's arm and the individualised last meal comprise some of the hidden moments of care, which tell us of the importance of these often hidden workers.
Parallel Session 2.1 - Lenses on building bereavement support, and building equitable care (Wednesday 3 November, $15: 30-16: 45)$

\section{0-5 ONLINE COMMUNITY OF PRACTICE DEVELOPMENT - PALLIATIVE CARE AND HOMELESSNESS}

1,2,3 Briony Hudson, ${ }^{2,3}$ Caroline Shulman. ${ }^{1}$ Marie Curie, London, UK; ${ }^{2}$ Marie Curie Palliative
Care Research Department, UCL, London, UK: ${ }^{3}$ Pathway, London, UK

\subsection{6/spcare-2021-Hospice. 5}

Background People experiencing homelessness have a high rate of most long-term conditions at a young age and are at high risk of dying young. Many people with complex and advanced physical and mental health problems are living in homeless hostels, and rarely have access to palliative care support. Hostel staff are often left to support people with inadequate health and social care input. In response, we created an online programme of learning to facilitate development of multidisciplinary communities of practice for people from different professional backgrounds in the same geographic location to connect.

Aims To develop and pilot a programme to:

1. Develop communities of practice to promote interprofessional working and support.

2. Develop shared understanding of palliative care and the different roles and services involved in the support of people experiencing homelessness.

3. Improve access to care for people experiencing homelessness with advanced ill health.

Learning from the pilot will be used to adapt the programme prior to national roll out.

Methods The programme, containing eight online sessions, was piloted in three locations. Topics relating to homelessness, inclusion health and palliative care were included. Sessions consisted of pre-recorded content and discussions focussing on shared learning and case discussions, acting as a vehicle for developing a multi-professional response to challenges encountered in real time. Data were collected via surveys and focus groups throughout, and after completion of sessions.

Results A range of homelessness, health and social care professionals attended the programme. Pilot data provided insight into the perceived usefulness of the programme in terms of connecting and empowering professionals and access to care and services. The importance of including a wide range of professional groups was highlighted and national roll out recommended.

Conclusion/Discussion To address the inequity that exists in palliative care access for people experiencing homelessness, a joined up, supportive, multi-professional response is essential. Online community of practice programmes are one way of achieving this. 